\title{
Intramedullary spinal cord abscess and subsequent granuloma formation: a rare complication of vertebral osteomyelitis detected by diffusion-weighted magnetic resonance imaging
}

\author{
Noriyoshi Takebe, M.D., Koichi Iwasaki, M.D., Ph.D., Hirokuni Hashikata, M.D., Ph.D., \\ and Hiroki Toda, M.D., Ph.D. \\ Department of Neurosurgery, Tazuke Kofukai Medical Research Institute and Kitano Hospital, Osaka, \\ Japan
}

An intramedullary spinal cord abscess, which is usually associated with discitis, is an uncommon but potentially important complication of vertebral osteomyelitis. The authors describe a rare case of an intramedullary conus medullaris abscess and lumbar osteomyelitis sparing the intervertebral discs and without discitis. The patient also developed a granuloma in the cauda equina during treatment. Diffusion-weighted MRI was useful for differentiating the granulomatous lesion from the relapse of infection.

A 65-year-old immunocompetent man with moderately controlled diabetes presented with progressive lowerextremity numbness and weakness with urinary dysfunction. He had progressive paraparesis, bilateral leg paresthesia, and sphincter compromise. Magnetic resonance imaging revealed an intramedullary ring-enhanced lesion, which was hyperintense on diffusion-weighted images. The lesion, an intramedullary spinal cord abscess, was surgically drained. During antibiotic treatment, serial MRI showed an enlarging enhanced lesion in the cauda equina, and a recurrent infection was suspected. A second-look surgery confirmed the formation of a granuloma and the absence of a relapse of the abscess. Although the enhanced lesion increased in size, its intensity on diffusion-weighted images remained unchanged. After 3 months of antibiotic treatment, all enhanced lesions were diminished.

An intramedullary spinal cord abscess is a rare but important complication of vertebral osteomyelitis, and it requires immediate treatment. Diffusion-weighted MRI was useful for the initial diagnosis as well as for monitoring treatment efficacy.

(http://thejns.org/doi/abs/10.3171/2014.6.FOCUS14141)

$\begin{array}{llll}\text { KEY WoRds } & \bullet & \text { intramedullary spinal cord abscess } \bullet \text { vertebral osteomyelitis } \\ \text { granuloma } & \bullet & \text { diffusion-weighted imaging } \bullet \quad \text { conus medullaris }\end{array}$

A $\mathrm{N}$ intramedullary spinal cord abscess is a known but uncommon complication of vertebral osteomyelitis; the number of reported patients with this association is small. ${ }^{2}$ Two previous studies have reported an intramedullary spinal cord abscess in patients with spondylodiscitis and epidural abscesses; ${ }^{3,5}$ however, this is the first reported case of an intramedullary spinal cord abscess in a patient with vertebral osteomyelitis in the absence of discitis or obvious epidural fluid collection. A unique feature of this infectious contagion is the possible hematogenous spread of microorganisms through the vascular network of the conus medullaris and cauda equina.

The diagnosis of intramedullary spinal cord abscess

Abbreviations used in this paper: $\mathrm{CRP}=\mathrm{C}$-reactive protein; $\mathrm{ESR}=$ erythrocyte sedimentation rate. is based on a high degree of suspicion that is raised by findings during patient examination and from diffusionweighted MRI.,9-11 Early diagnosis is essential to minimize the neurological deficits, and immediate treatment enhances the chance of recovery. ${ }^{1}$ In the current case, a granuloma formed after abscess drainage and during antibiotic treatment. Diffusion-weighted imaging was useful for differentiating the inflammatory reaction from a relapse of infection.

\section{Case Report}

History. Two months before admission to our hospital, a diabetic 65-year-old Japanese man experienced left thigh pain. After 6 weeks of gradually worsening pain, he had a local orthopedic consultation. Thereafter, he developed 
urinary incontinence and bilateral lower-leg numbness extending from his thighs to the soles of his feet. Five days later, he was unable to walk because of the weakness and numbness in his legs. In addition, he had saddle anesthesia, was not able to control urinary voiding, and had bowel and bladder incontinence. He had been diagnosed with a spinal tumor at a local hospital, and he had been treated with high-dose intravenous steroids for 3 days. Subsequently, he was transferred to our institute for further treatment. He was immunocompetent, and his diabetes was moderately controlled. In addition, he had a history of hypertension, myocardial infarction, benign prostatic hyperplasia, and degenerative lumbar spondylosis.

Examination. On neurological examination, we found that the patient was distressed by his leg pain and weakness, but he was alert and oriented. He had flaccid paraparesis. Power was reduced in both lower extremities. His Medical Research Council grades were 3/5 for the left iliopsoas and quadriceps muscles, $4 / 5$ for the right iliopsoas and quadriceps muscles, and 3/5 for the bilateral hamstrings, anterior tibialis, extensor hallucis longus, and gastrocnemius muscles. Knee-jerk and ankle-jerk reflexes were absent on both sides. Anal tone was slightly lax. There was significant hypesthesia to thermal and painful nociception and light touch sensation below the level of the left L-1 and right L-2 segments, including the perianal area. Proprioception of the bilateral ankle joints and his sense of vibration were spared. His neck was supple and did not show Kernig's or Brudzinski's signs. Examination of the cranial nerves and upper limbs yielded unremarkable findings. The Japanese Orthopaedic Association score for low-back pain was 4 of 29.

Laboratory results showed a white blood cell count of 8300 cells $/ \mathrm{mm}^{3}$ with $84 \%$ neutrophils, $1.5 \mathrm{mg} / \mathrm{dl} \mathrm{C}$ reactive protein $(\mathrm{CRP})$, an erythrocyte sedimentation rate (ESR) of $60 \mathrm{~mm} / \mathrm{min}$, and a hemoglobin A1c level of $7.6 \%$. A serological test for human immunodeficiency virus was negative. Protein and glucose levels in the CSF were $367.3 \mathrm{mg} / \mathrm{dl}$ and $137 \mathrm{mg} / \mathrm{dl}$, respectively, with 343 mononuclear and 5 polynuclear white blood cells. A test for tuberculosis and a microbiological culture of blood and CSF were negative.

Lumbar spine radiography and CT showed mild osteolysis of the dorsal part of the L-1 and L-2 vertebral bodies. Contrast-enhanced MRI showed hyperintense changes on T1- and T2-weighted images of the L-1 and L-2 vertebral bodies (Fig. 1A and B). The lumbosacral spinal cord was swollen and hyperintense on T2-weighted images (Fig. 1A), and the lesion was ring enhanced (Fig. 1B), which was hyperintense on diffusion-weighted images (Fig. 1C). The intervertebral disc intensity was neither diminished nor enhanced, and there was no fluid accumulation in the epidural space.

Operation. With a preoperative diagnosis of intramedullary spinal cord abscess, the patient underwent T11L1 laminectomy. Upon opening the dura and arachnoid membrane, the underlying conus medullaris was found to be swollen, and an exophytic mass was noted caudal to this structure (Fig. 2A). Opening the capsule drained the purulent discharge (Fig. 2B), from which methicillin-sen- sitive Staphylococcus aureus was isolated. The abscess cavity was completely irrigated with tobramycin solution. Subsequently, a 4-g dose of intravenous ceftriaxone was administered daily, with an additional vancomycin dosage for the first 4 days. Ceftriaxone was given for 36 days, and this was followed by a daily dose of $3 \mathrm{~g}$ cefmetazole and $1.2 \mathrm{~g}$ clindamycin.

Postoperative Course. After 2 weeks of the treatment, the enhanced intradural mass involving the cauda equina at the L1-2 level increased in size, although the ring-enhanced lesion had decreased in size (Fig. 3A and B). Although the ESR and CRP levels, as well as the image intensity of the lesion on diffusion-weighted images (Fig. 3E-G), remained unchanged (Fig. 3H), the enhanced mass grew in size (Fig. 3C), and the patient underwent second-look surgery to confirm the recurrence of the abscess. We found the enhanced lesion to be a granuloma without purulent discharge (Fig. 2C). Therefore, we continued cefmetazole and clindamycin therapies for 6 and 7 weeks, respectively, until the ESR and CPR levels returned to within normal limits (Fig. 3H). Magnetic resonance imaging, performed 9 months postoperatively, showed the lesion in the cauda equina to be less enhanced and smaller (Fig. 3D). The lesion was not significantly hyperintense on diffusion-weighted imaging (Fig. 3G). On examination 12 months postoperatively, the patient could walk without assistance, and his impaired muscle strength had returned to normal except in the right anterior tibialis, which showed an Medical Research Council grade of 4/5. Mild bilateral lower foot dysesthesia persisted. He could control defecation but needed intermittent self-catheterization for urination. His Japanese Orthopaedic Association score 12 months after treatment was 15 of 29 , which was an improvement of $44 \%$.

\section{Discussion}

Intramedullary spinal cord abscesses, which are rare, especially in the antibiotic era ${ }^{2}$ form a potentially serious complication of pyogenic vertebral osteomyelitis. The 2 cases that have been reported previously ${ }^{3,5}$ involved combined infections related to an intramedullary spinal cord abscess and spondylodiscitis with an epidural abscess. In the current case, the patient, who was diabetic but immunocompetent, exhibited osteomyelitis of 2 adjacent vertebral bodies and an infection of the caudal end of the spinal cord. The vertebral endplate was not destroyed, and the intervening disc was not infected. There was no epidural fluid collection, subdural empyema, or meningitis. To the best of our knowledge, this unique infectious pattern has not been reported previously.

Spinal infections usually spread through the hematogenous and contiguous pathways and rarely through the lymphatic route. ${ }^{1,6}$ Our patient presented with diabetes and was at a high risk of infection. He might have had an infection of unknown origin and then developed both vertebral osteomyelitis and an intramedullary spinal cord abscess through the hematogenous routes of infectious spread. Hematogenous routes can be arterial paths to adjacent vertebral bodies and to the conus medullaris through the segmental lumbar artery and anterior and 


\section{Intramedullary spinal cord abscess and vertebral osteomyelitis}
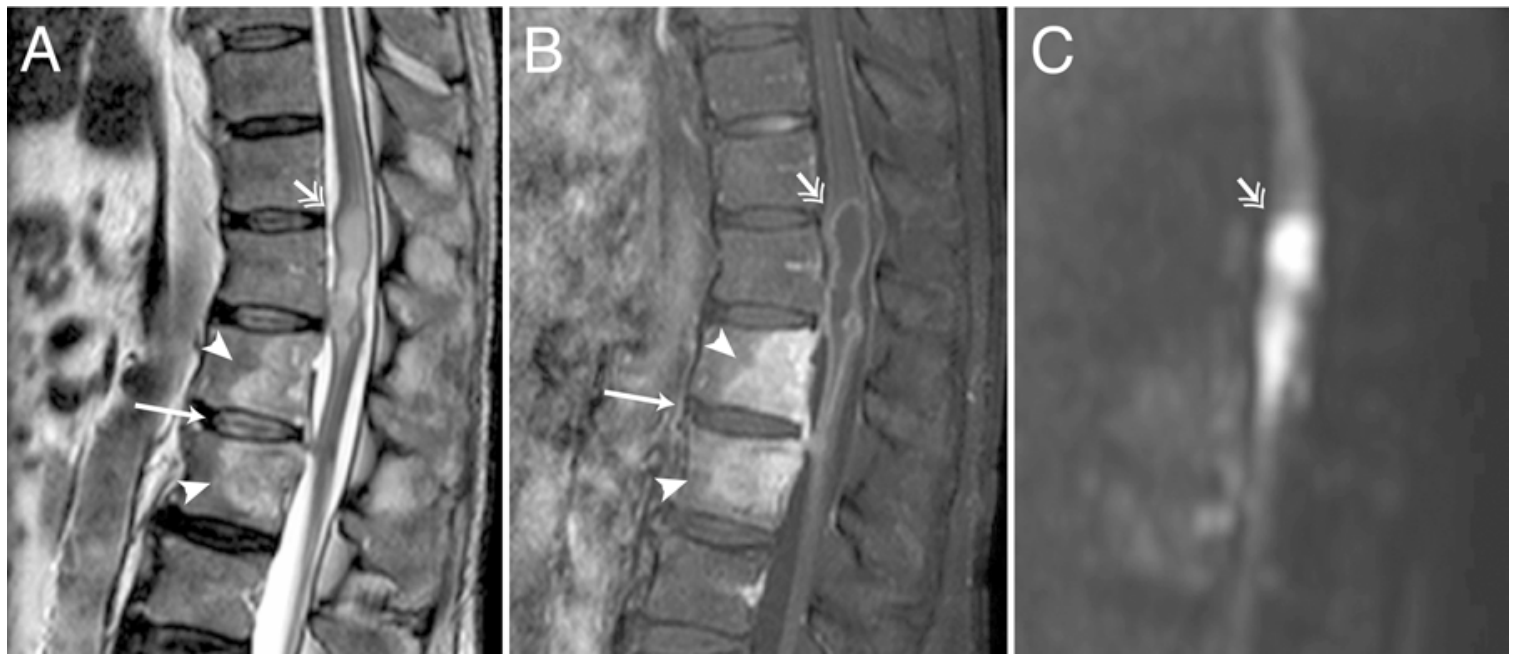

FIG. 1. Preoperative sagittal T2-weighted (A), T1-weighted Gd-enhanced (B), and diffusion-weighted (C) MR images showing osteomyelitis of the L1-2 vertebral bodies (arrowheads) and a ring-enhanced intramedullary abscess (2-headed arrows) extending from the T-11 to the T-12 level to the cauda equina. The endplates are intact without obvious intervening disc space infection (arrows), epidural fluid collection, or subdural empyema.

posterior radicular arteries, which are downstream from the segmental lumbar artery. ${ }^{4}$ Infection can also spread from the basivertebral vein and the anterior internal vertebral venous plexus to the segmental vein and its radicular vein as well as to the terminal vein of the cauda equina through the venous system. ${ }^{12}$ Vertebral osteomyelitis has often been associated with disc space infection and epidural abscesses. ${ }^{8}$ In the current case, we were not able to find an epidural fluid collection or a disc space infection, which distinguished the current case from other cases. Our patient might have become symptomatic prior to disc space infection due to the development of an intramedullary spinal cord abscess.

Early diagnosis and prompt treatment, including surgical approaches, for intramedullary spinal abscesses are important to minimize sequelae. ${ }^{1}$ Diagnosis is sometimes difficult, and recent studies have reported the usefulness of diffusion-weighted MRI., ${ }^{79-11}$ In the current case, the patient had granuloma formation after the initial surgi$\mathrm{cal}$ and antibiotic treatments. An accurate recurrence rate for spinal infection cannot be determined, and treatment failure is often reported in cases of spondylodiscitis. ${ }^{8}$ Our findings showed that analysis of intensities of diffusionweighted images could form a useful index with which to evaluate the infectious status and treatment efficacy for lesions that are suspected to be a relapse of an abscess based on the enlargement of an enhanced lesion.

\section{Conclusions}

In summary, this case demonstrated the rare association of an intramedullary abscess with vertebral osteomyelitis for which surgical drainage of the abscess was effective in combination with appropriate antibiotic treatment. Serial evaluation with diffusion-weighted imaging is useful to monitor treatment efficacy and to differentiate noninfectious inflammatory reactions from relapses of infection as well as for the initial diagnosis of abscesses. The combination of vertebral osteomyelitis and an intramedullary spinal cord abscess without disc space infection represents a unique pattern of spinal infection, but it should be recognized that these disorders require urgent treatment.
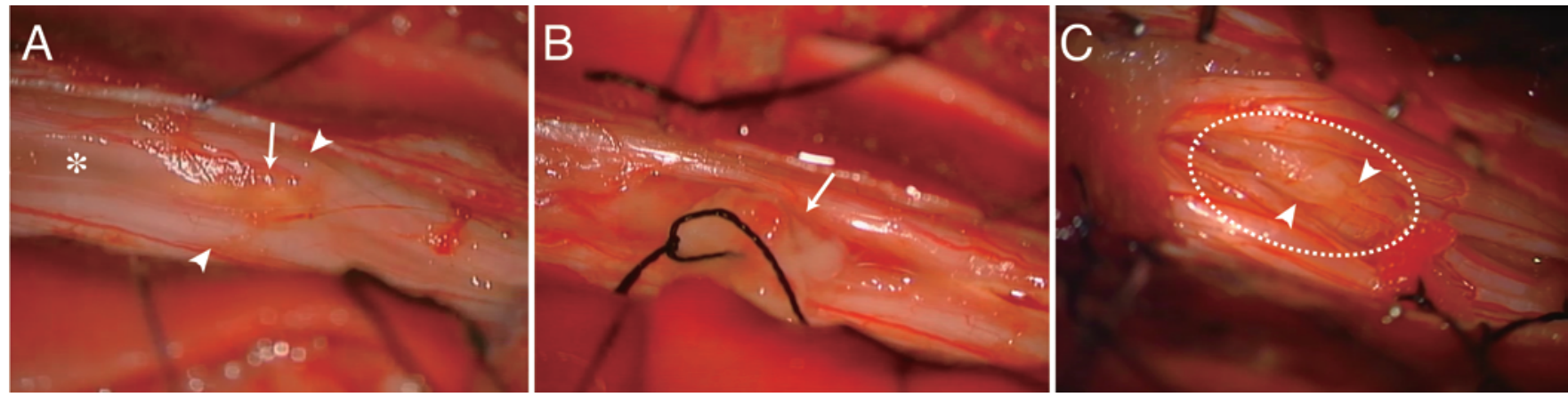

FIG. 2. A: Intraoperative photograph of the first operation showing the swollen soft mass (arrow) in the caudal end of the conus medullaris (asterisk) between the roots of the cauda equina (arrowheads), which are covered with slightly thickened arachnoid. B: Intraoperative photograph. Opening the wall of the soft mass drains the purulent discharge (arrow). C: Intraoperative photograph. At the second operation, a moderately hard granuloma (dotted circle) was found that involved the roots of the cauda equina (arrowheads). 
postoperative Gd-enhanced diffusion-weighted

days

9
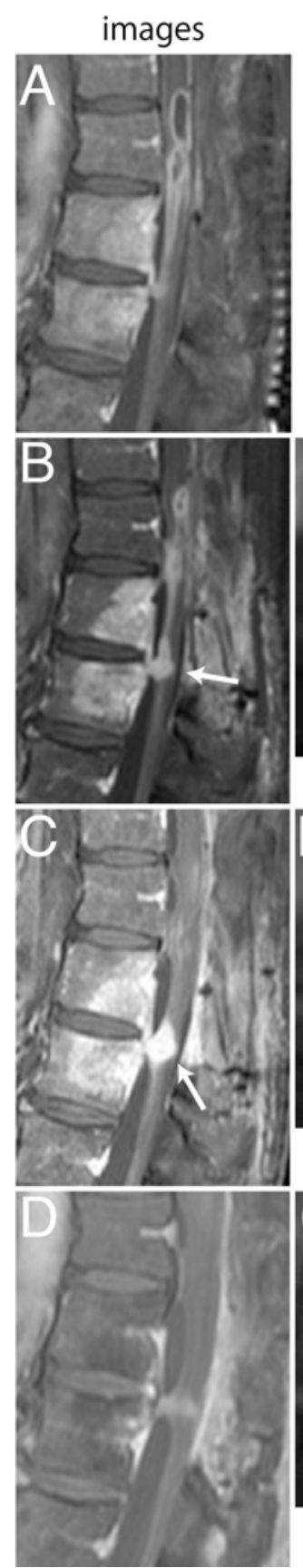

images
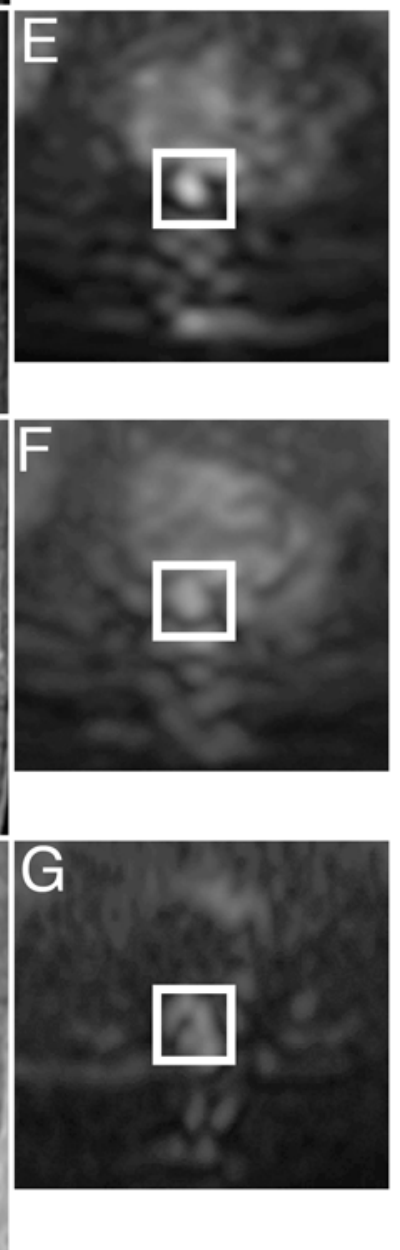

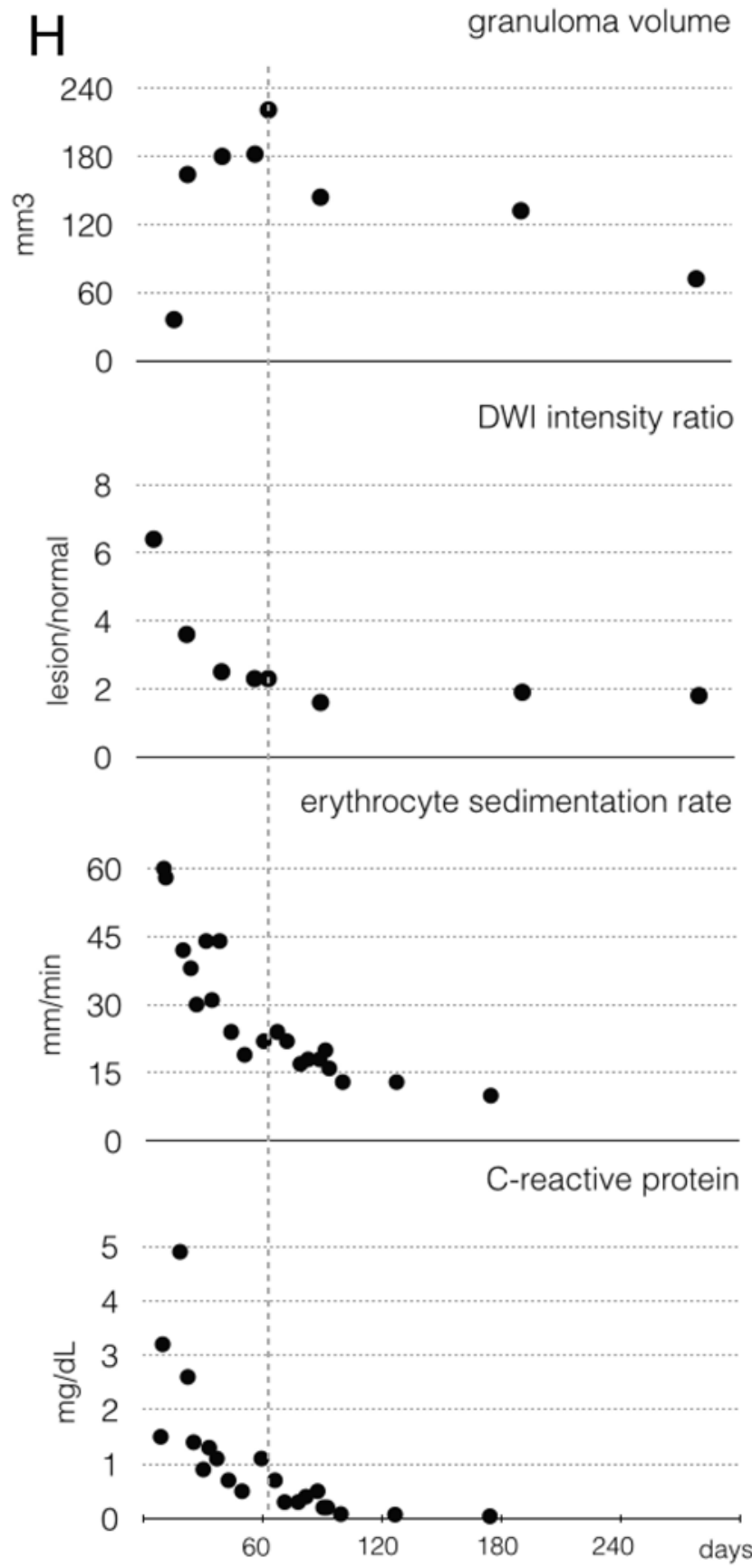

FIG. 3. A-D: Serial postoperative sagittal T1-weighted Gd-enhanced images showing the formation of the granuloma during the treatment (arrows, B and C). E-G: The image intensity of the Gd-enhanced lesion (squares) is measured on the diffusionweighted images. H: The serial granuloma size, the diffusion-weighted imaging (DWI) intensity ratio, the values of the ESR, and the levels of CRP are plotted along the clinical time course. The gray vertical dashed line indicates the day when the enhanced lesion showed the maximal increase (C). The image intensity on diffusion-weighted images of the lesion did not increase at the same time (F).

\section{Disclosure}

The authors report no conflict of interest concerning the materials or methods used in this study or the findings specified in this paper.

Author contributions to the study and manuscript preparation include the following. Conception and design: Toda, Takebe. Acquisition of data: Toda, Takebe. Analysis and interpretation of data:
Toda, Takebe. Drafting the article: Toda, Takebe. Critically revising the article: Toda, Iwasaki, Hashikata. Reviewed submitted version of manuscript: all authors. Approved the final version of the manuscript on behalf of all authors: Toda. Study supervision: Toda, Iwasaki.

\section{References}

1. Byrne RW, von Roenn KA, Whisler WW: Intramedullary 


\section{Intramedullary spinal cord abscess and vertebral osteomyelitis}

abscess: a report of two cases and a review of the literature. Neurosurgery 35:321-326, 1994

2. Chan CT, Gold WL: Intramedullary abscess of the spinal cord in the antibiotic era: clinical features, microbial etiologies, trends in pathogenesis, and outcomes. Clin Infect Dis 27:619626, 1998

3. Crema MD, Pradel C, Marra MD, Arrivé L, Tubiana JM: Intramedullary spinal cord abscess complicating thoracic spondylodiscitis caused by Bacteroides fragilis. Skeletal Radiol 36:681-683, 2007

4. Crock HV, Yamagishi M, Crock MC: The Conus Medullaris and Cauda Equina in Man: An Atlas of the Arteries and Veins. New York: Springer-Verlag, 1986, pp 2-47

5. Derkinderen P, Duval X, Bruneel F, Laissy JP, Regnier B: Intramedullary spinal cord abscess associated with cervical spondylodiskitis and epidural abscess. Scand J Infect Dis 30:618-619, 1998

6. DiTullio MV Jr: Intramedullary spinal abscess: a case report with a review of 53 previously described cases. Surg Neurol 7:351-354, 1977

7. Dörflinger-Hejlek E, Kirsch EC, Reiter H, Opravil M, Kaim AH: Diffusion-weighted MR imaging of intramedullary spinal cord abscess. AJNR Am J Neuroradiol 31:1651-1652, 2010

8. Gouliouris T, Aliyu SH, Brown NM: Spondylodiscitis: update on diagnosis and management. J Antimicrob Chemother 65 (Suppl 3):iii11-iii24, 2010
9. Hood B, Wolfe SQ, Trivedi RA, Rajadhyaksha C, Green B: Intramedullary abscess of the cervical spinal cord in an otherwise healthy man. World Neurosurg 76:361.e15-361.e19, 2011

10. Iwasaki M, Yano S, Aoyama T, Hida K, Iwasaki Y: Acute onset intramedullary spinal cord abscess with spinal artery occlusion: a case report and review. Eur Spine J 20 (Suppl 2): S294-S301, 2011

11. Roh JE, Lee SY, Cha SH, Cho BS, Jeon MH, Kang MH: Sequential magnetic resonance imaging finding of intramedullary spinal cord abscess including diffusion weighted image: a case report. Korean J Radiol 12:241-246, 2011

12. Thron AK: Vascular Anatomy of the Spinal Cord: Neuroradiological Investigations and Clinical Syndromes. Vienna: Springer-Verlag, 1988, pp 39-58

Manuscript submitted April 15, 2014.

Accepted June 10, 2014.

Please include this information when citing this paper: DOI: 10.3171/2014.6.FOCUS14141.

Address correspondence to: Hiroki Toda, M.D., Ph.D., 2-4-20, Ohgimachi, Kita, Osaka 530-8480, Japan. email: hi-toda@kitanohp.or.jp. 